\title{
Coalescence of Microscopic Polymeric Drops: Effect of Drop Impact Velocities - Supporting Information
}

\author{
Vishal Sankar Sivasankar, ${ }^{1}$ Sai Ankit Etha, ${ }^{1}$ Daniel R. Hines, ${ }^{2}$ and Siddhartha Das ${ }^{1 *}$ \\ ${ }^{1}$ Department of Mechanical Engineering, University of Maryland, College Park, MD 20742 \\ ${ }^{2}$ Laboratory for Physical Sciences, 8050 Greenmead Drive, College Park, Maryland 20740, \\ United States
}

*sidd@umd.edu 
Here we show the distribution of the magnitude of the non-dimensional velocity gradient $\nabla^{*} U^{*}$ $\left(=\frac{\nabla U}{U_{0} / R}\right)$ inside and around the droplets at various instances during the coalescence of the droplets for a Weber number of $\mathrm{We}=0$. In Fig. A1, we observe that the shear rate (or the velocity gradient) is higher at times immediately after the impact which will lead to lower effective viscosity. However, we observe a much smaller shear rates at later stages of spreading leading to a higher viscosity at these times. Such a behavior is typical in the spreading of shear-thinning liquid. ${ }^{1}$ 
(a) $\mathrm{t}^{*}=99.76$

(i)

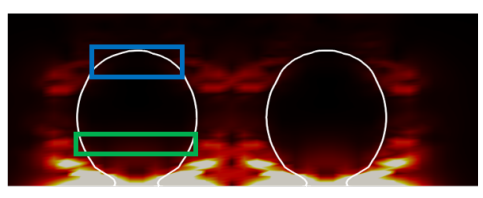

(b) $t^{*}=105.31$

(i)

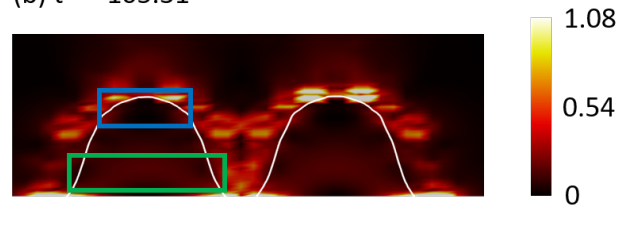

(c) $t^{*}=109.19$

(i)

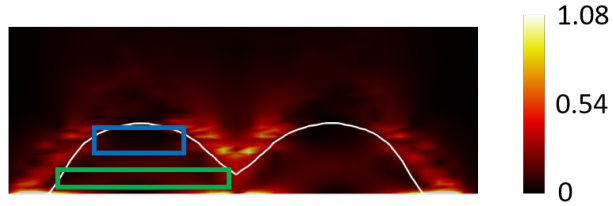

(d) $t^{*}=138.56$

(i)

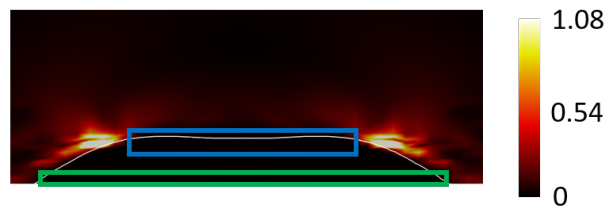

$\left|\nabla^{*} U^{*}\right|$

1.8

1.44

0.72

0

$\left|\nabla^{*} U^{*}\right|$

.08

.54

$\nabla^{*} U^{*} \mid$

.08

4

$\left|\nabla^{*} U^{*}\right|$

1.08

0.54 (ii)

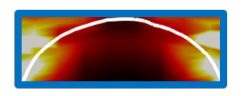

(iii)

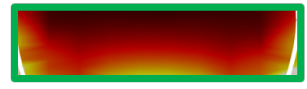

(ii)

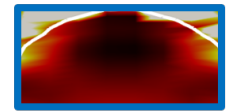

(iii)

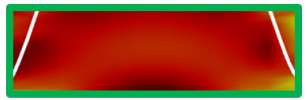

(ii)

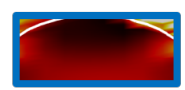

(iii)

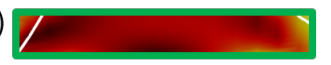

(ii)

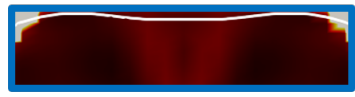

(iii) $\left|\nabla^{*} U^{*}\right|$

0.18

0.14

0.07

0

$\left|\nabla^{*} U^{*}\right|$

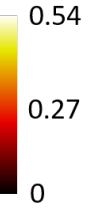

$\left|\nabla^{*} U^{*}\right|$

0.43

0.22

$\left|\nabla^{*} U^{*}\right|$
0.05

0.025

Figure S1: Variation of the magnitude of dimensionless velocity gradient $\left(\left|\nabla^{*} U^{*}\right|\right)$ inside two polymeric and power-law obeying (with $\mathrm{n}=0.6$ ) drops of radii $10 \mu \mathrm{m}$ as well as inside the air in the immediate vicinity of the drops across a cross-section (i.e., the y-z plane) that passes through center of the drops $(\mathrm{x}=0)$ at different time instants (dimensionless) inside the coalescing drops for $W e=0$. Here the dimensionless time is $t^{*}=\frac{t}{t_{c}}$, the dimensionless velocity gradient is $\nabla^{*} U^{*}=$ $R \nabla\left(U / U_{0}\right)$ (where $\mathrm{U}_{0}=\mathrm{R} / \mathrm{t}_{\mathrm{c}}$ is the characteristic velocity scale), and $t_{c}=\sqrt{\rho_{1} \mathrm{R}^{3} / \sigma}$ is the capillary time scale. Here, for different time instances, we show distribution of the gradient over the entire drop in (i) and provide magnified views at locations near the top and bottom of the drop in (ii) and (iii) respectively. 


\section{References}

1. Dechelette, A.; Sojka, P. E.; Wassgren, C. R. Non-Newtonian Drops Spreading on a Flat Surface. J. Fluid. Eng. 2010, 132, 101302. 